\title{
Three-Dimensional Design for Buttock Augmentation: The BACVA Approach
}

\section{Vallarta Rodriguez $A^{1 *}$, Rojas Garcia $\mathrm{P}^{2}$, Ruiz Treviño ${ }^{3}$, Guerrero Burgos $\mathrm{F}^{5}$, Euan Vazquez $\mathrm{C}^{2}$, Chavez Osorio $\mathrm{F}^{2}$, Guerra Rendon $\mathrm{D}^{4}$ and Villaseñor Villalpando $\mathrm{E}^{4}$}

1Plastic and Reconstructive Surgeon, Professor of plastic surgery course at Central Hospital of High Specialty (PEMEX) / Universidad Nacional Autónoma de México, Mexico

${ }^{2}$ General Surgeon, Resident at Central Hospital of High Specialty Plastic and Reconstructive Surgery (PEMEX), Mexico ${ }^{3}$ Advanced Minimally Invasive Surgeon, Plastic and Reconstructive Surgeon, Mexico

${ }^{4}$ Resident at Central Hospital of High Specialty Plastic and Reconstructive Surgery (PEMEX), Mexico ${ }^{5}$ Plastic and Reconstructive Surgeon, Mexico

*Corresponding author: Vallarta Rodriguez A, Puente de Piedra 150-419 T 2, Ciudad de México, CP 14050, Mexico, Tel: 5255 55284489; Email: avallarta1@gmail.com

\section{Abstract}

Autologous augmentation by lipografts has become the procedure of choice for enhancing the buttock area for many plastic surgeons. It avoids the complications of prosthetic materials while taking advantage of tissue interactions between grafts and receptor tissues. It has the added benefit of combining a body defining lipoplasty, offering a faster recovery and less long-term complications compared to prosthetic implant augmentation. Objectives: The authors present a new surgical approach for buttock augmentation / reshaping with lipografts. By implementing a systemized, mathematic-based approach for delivering controlled 3D volumes to our practice, creating a parabolic design similar to a gluteal implant, we can obtain predictable and permanent results. Methods: Between 2008 and 2013, a group of 75 patients underwent autologous augmentation by lipografts for buttock volume and reshaping by dividing each buttock into nine quadrants. Adhering to the aesthetic ideal proportions, exact percentages of volume are delivered, crafting a beautiful and natural buttock in relation to the patient's own anatomy. Results: 75 female patients between 24 and 52 years underwent buttock augmentation with the BACVA technique. All patients experienced favorable convalescence with $94 \%$ patient satisfaction rate. 9 patients (12\%) underwent ambulatory seroma aspiration with complete resolution. There were no mayor complications in this series. Conclusions: The authors have demonstrated that using BACVA 


\section{International Journal of Transplantation \& Plastic Surgery}

predictable, natural, beautiful and permanent results may be achieved. It is a mathematic-based approach that is reproducible and easy for the plastic surgeon in training to master. Level of Evidence: 5.

Keywords: Buttock augmentation; Buttock reshaping; Gluteal augmentation; Lipograft; Lipoinjection; structured lipografting; Fat transplantation

\section{Introduction}

Gluteal augmentation has become a popular procedure in plastic surgery. Although the ideal of beauty varies among cultures, most agree that the attractive feminine shape consists of a narrow waist and full buttocks (the so called "hourglass silhouette") [1,2]. The ideally beautiful buttock is defined as a waist to hip ratio (WHR) near 0.7. ${ }^{3}$ This concept must be taken in consideration when performing a buttock reshaping procedure because a successful outcome depends on bringing the patient as close to the ideal WHR as posible $[3,4]$. Superficial landmarks useful in defining a beautiful and youthful buttocks are the two medial sacral crest dimples corresponding to the posterior-superior iliac spines (PSIS), the V-shaped sacral triangle and the infragluteal folds that do not extend beyond the medial third of each thigh $[5,6]$. The point of maximum gluteal projection should correspond to the level of the mons pubis [5].

Traditional ideal aesthetic buttock proportions can be correctly applied to the feminine patient and easily achieved by incorporating this new method named The Buttock Augmentation with Controlled Volumetric Approach (BACVA), offering a parabolic design similar to a gluteal implant which provides reliable, reproducible and safe results.

\section{Methods}

75 female patients were treated with this method. After marking the areas to be lipoaspirated, we designed a nine-quadrant pattern by dividing each buttock into thirds in a vertical and horizontal direction, resembling a wind rose. The central zone was considered the point of maximum projection and it is where $50 \%$ of the total volume of lipografts were transferred. We defined, 4 primary and 4 minor zones. The first 4 zones represented the areas along the cardinal axes, and received $40 \%$ of the total volume. The latter 4 zones, corresponded to the quadrants located in the diagonal axes and held the remaining $10 \%$ of the volume (Figure 1, Supplemental Digital Content 1).

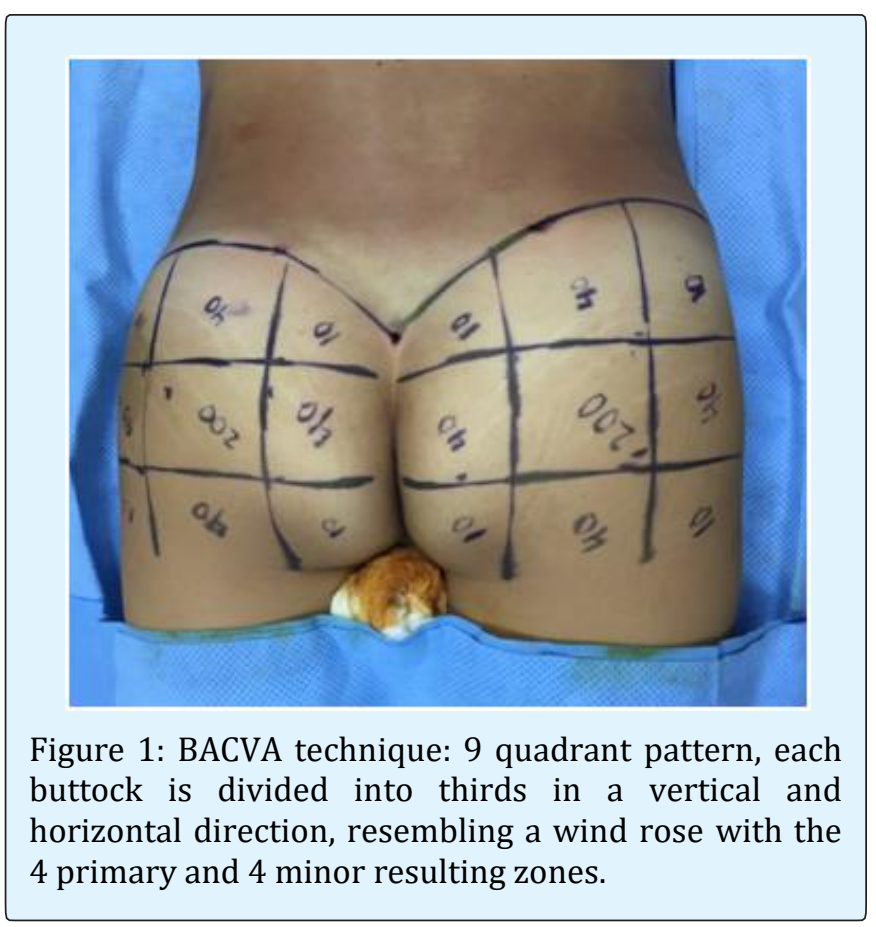

General anesthesia was administered and skin was prepped with povidone-iodine solution. The genital and anal areas were isolated with povidone-soaked pads. All patients received a preoperative dose of cefalotin (unless allergic) plus clindamycin. After infiltrating normal saline wetting solution with epinephrine $(1: 500,000)$ and no additional lidocaine and waiting 15 minutes, traditional lipoaspiration was done in previously marked areas. The initial lipoplasty/fat grafting procedure was performed either with a $60 \mathrm{~mL}$ syringe or a lipoaspirator set on low power. The lipoaspirate was left to settle by gravity and gentle filtration, avoiding any traumatic processing. Formerly we transferred the lipografts with the same $60 \mathrm{~mL}$ syringes and $3 \mathrm{~mm}$ cannulas at the beginning, but we modified our technique incorporating $10 \mathrm{~mL}$ syringes and using $2 \mathrm{~mm}$ atraumatic cannulas.Lipografts were delivered in a controlled fashion with small 2-3 $\mathrm{mm}$ retrograde movements, distributing themin different planes from deep to superficial and from caudal to 


\section{International Journal of Transplantation \& Plastic Surgery}

cephalic subcutaneous tissues, forming a threedimensional grid.

This procedure was performed on each buttock as needed until desired shape and symmetry were achieved (see Video, Supplemental Digital Content 2, which demonstrates the BACVA technique). We systematically applied a firm massaging motion to each buttock when needed in order to homogenize any remaining irregularities.

After lipografting, when extensive liposuction of the back was performed, we consistently left close suction drains above the buttock area. This strategy aimed to isolate the lipografts from the back's drainage, in order to reduce the possibility of buttocks' infection or collection. Drains were removed once serous drainage was reported less than $30 \mathrm{cc}$ in 24 hours. Incisions were closed with 4-0 non-absorbable sutures. Immediate compression garments were indicated in every patient over lipoaspirated areas. The buttocks remain edgarment free, reducing the possibility of compression ischemia.

All patients were hospitalized for 24 hours, and had apostoperative full blood count. Oral antibiotic (a first generation cephalosporin + clindamycin) and analgesic medications (non-narcotic) were prescribed for a week. Immediate movilization was encouraged. Compression garnments were indicated for 6 weeks.

\section{Results}

75 female patients were treated with BACVA. Male patients were excluded from this study. Patients' age ranged from 24 to 52 years (median 34 years).

Surgical time ranged from 90 to 180 minutes (average 135 minutes). The total lipoaspirate ranged from 2.0 to 4.0 liters. The total volume of transplanted lipografts ranged from $150 \mathrm{cc}$ to $400 \mathrm{cc}$ (average of $300 \mathrm{cc}$ ) per buttock. The average follow up time was 1.5 years.

9 patients (12\%) presented seroma formation, all of them were treatedwith office aspiration. 15 patients (20\%) required red blood cell transfusion after a full blood count.There were no major complications. Pain control was adequate with oral non-opioid analgesics. During followup visits, patients were inquired ontheir overall satisfaction rate on a scale from zero to one hundred, with a 94\% esthetic satisfaction after buttock augmentation / reshaping using the BACVA approach (Figure 2, Supplemental Digital Content 3).

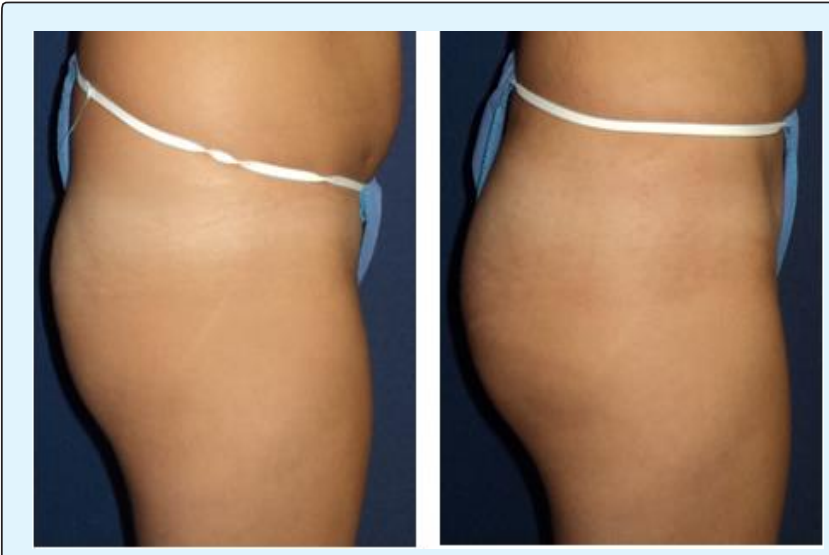

Figure 2: Pre and postoperative images. Female patient with 300cc of lipoinjection with BACVA.

\section{Discussion}

Throughout the years surgeons have relied mainly on three procedures to increase buttock size: autologous augmentation by lipoinjection, silicon implants and muscle flaps [6-8]. Autologous lipoinjection has gained popularity in the last decade becoming the method of choice for many plastic surgeons. It has the added benefit of a body defining lipoplasty. Autologous augmentation by lipografts offers a faster recovery and less long-term complications compared to augmentation with silicone implants [9-15].

Most scientific papers on buttock augmentation by lipoinjection focus on fat grafting techniques, fat handling and processing, lipotransfer techniques, and planes for lipografts placement [9-18]. Nevertheless they do not describe a predictable, mathematic-based method for delivering lipografts in adequately distributed volumes that can reproduce the 3 dimensional parabolic design of the gluteal implant anatomy and solely rely on the surgeon's intuition or impression. This makes reproducibility difficult to evaluate and a steeper learning curve for in-training plastic surgeons. We share a mathematic-based method to obtain consistent and longlasting results by applying volume strategically throughout quadrants that has given us gratifying results.

The BACVA (Buttock Augmentation with Controlled Volumetric Approach) was designed based on the ideal buttock shape, taking into account a gluteal implant's parabolic architecture, which resembles a wind sail. In order to achieve this design, we planned a grid with the 9 areas of lipograft distribution described before as: 


\section{International Journal of Transplantation \& Plastic Surgery}

1 central zone, considered the point of maximum projection, where $50 \%$ of the total volume of lipografts must be transferred primary and 4 minor zones. The primary zones run along the cardinal axes, and should receive $40 \%$ of the total volume. The minor zones, belong to the quadrants located in the diagonal axes and receiving the remaining $10 \%$ of the volume.

When considering this approach one must have in mind that that this model cannot be applied to every patient, we must make a complete anatomic evaluation, taking into account the areas that need to be augmented. And we suggest that at least $300 \mathrm{cc}$ to $350 \mathrm{cc}$ of pure lipoaspirate must be obtained (excluding any wetting solution). If these basic principles are not met, the aesthetic objective is better achieved by silicone implant augmentation or other body contour techniques. This is especially important in thin patients.

An alternative volume distribution can be achieved in patients seeking enhanced projection and lateral fullness: $60 \%$ of the volume is transferred in the central zone and the reminding $40 \%$ in peripheral zones, always trying to maintain a 4:1 relation in primary zones to minor zones. We noticed a drop in seroma formation after incorporating the use of drains as previously described. Our team has a low threshold for transfusing erythrocyte concentrates when the postoperative control hemoglobin level is below $9.0 \mathrm{mg} / \mathrm{dL}$. We did not find any association between blood transfusion and body mass index.

The BACVA approach allows us to selectively modify all of the nine zones previously described with precise volume distribution. Its three-dimensional structure must resemble a grid with ribbons of lipografts 2 to $3 \mathrm{~mm}$ apart, allowing adequate nourishment and integration of the grafts. This design permits a natural and permanent result. BACVA offers a mathematic-based alternative to traditional lipoinjection techniques. It not only permits augmentation, it enables the surgeon to truly reshape the buttocksof selected patients. The versatility in its design permits adaptations in order to meet surgeons preferences and deliver personalized and unique results. This new logic-based approach enhances the learning experience of residents and fellow plastic surgeons positively, making the procedure easier to master.

\section{Conclusion}

Aesthetic buttock augmentation/reshaping must be a safe procedure with consistent and reproducible outcomes. By delivering planned and controlled volumes three-dimensionally, an aesthetically ideal buttock can be created. In our hands, BACVA approach is an attractive option for autologous buttock reshaping in selected female patients, offering predictable, consistent, beautiful and permanent results. Traditional ideal aesthetic buttock proportions can be correctly applied to the feminine patient and easily achieved by incorporating the described method of buttock contouring [1-3].

\section{References}

1. Singh D (2006) Universal Allure of the Hourglass Figure: An Evolutionary Theory of Female Physical Attractiveness. Clin Plastic Surg 33(3): 359-370.

2. Lee EI, Roberts TL, Bruner TW (2009) Terrence WB. Ethnic Considerations in Buttock Aesthetics. Semin Plast Surg 23(3): 232-244.

3. Singh D (1993) Adaptive significance of female physical attractiveness: role of waist-to-hip ratio. J Pers Psychol Soc Psychol 65(2): 293-307.

4. Cuenca-Guerra R, Lugo-Beltran I (2006) Beautiful buttocks: characteristics and surgical techniques. Clin Plast Surg 33(3): 321-32.

5. Raul Gonzalez (2006) Etiology, Definition, and Classification of Gluteal Ptosis. Aesth Plast Surg 30(3): 320-326.

6. Centeno RF, Young VL (2006) Clinical Anatomy in Aesthetic Gluteal Body Contouring Surgery. Clin Plastic Surg 33(3): 347-358.

7. De la Peña JA, Rubio OV, Cano JP, Cedillo MC, Garcés MT (2006) History of gluteal augmentation. Clin Plast Surg 33(3): 307-319.

8. Constantino GM (2003) Gluteoplasty. Aesthetic Surg J 23(6): 441-455.

9. Roberts TL 3rd1, Weinfeld AB, Bruner TW, Nguyen K (2006) "Universal" and Ethnic Ideals of Beautiful Buttocks are Best Obtained by Autologous Micro Fat Grafting and Liposuction. Clin Plastic Surg 33(3): 371394.

10. Roberts TL 3rd, Toledo LS, Badin AZ (2001) Augmentation of the Buttocks by Micro Fat Grafting. Aesthetic Surg J 21(4): 311-319.

11. Aiache AE (2006) Gluteal re-contouring with combination treatments: implants, liposuction, and fat transfer. Clin Plast Surg 33(3): 395-403. 


\section{International Journal of Transplantation \& Plastic Surgery}

12. Juan Carlos Cardenas Restrepo, Jaime Alberto Muñoz Ahmed (2002) Large-volume Lipoinjection for Gluteal Augmentation. Aesthetic Surg J 22(1): 33-38.

13. Ercan Karacaoglu, Nazim Durak (2012) A fifteen-year experience of buttock contouring with combination of silicone implant, liposuction, and structural fat grafting. Eur J Plast Surg 35(1): 81-87.

14. Cárdenas-Camarena L, Silva-Gavarrete JF, ArenasQuintana R (2011) Gluteal Contour Improvement: Different Surgical Alternatives. Aesth Plast Surg 35(6): 1117-1125.

15. Nicareta B, Pereira LH, Sterodimas A, Illouz YG (2011) Autologous Gluteal Lipograft. Aesth Plast Surg 35(2): 216-224.
16. Murillo WL (2004) Buttock Augmentation: Case Studies of Fat Injection Monitored by Magnetic Resonance Imaging. Plast Reconstr Surg 114(6): 1606-1614.

17. Herold C, Ueberreiter K, Busche MN, Vogt PM (2013) Autologous Fat Transplantation: Volumetric Tools for Estimation of Volume Survival. A Systematic Review. Aesth Plast Surg 37: 380-387.

18. Frank SJ, Flusberg M, Friedman S, Sternschein M, Wolf EL (2014) Aesthetic surgery of the buttocks: imaging appearance. Skeletal Radiol 43: 133-139. 